\title{
Reproductive coercion and contraceptive use in Ethiopia
}

\author{
Neetu A. John and Jeffrey Edmeades \\ International Center for Research on Women \\ Washington DC \\ njohn@icrw.org
}

\begin{abstract}
Context: While intimate partner violence (IPV) is recognized as a major contributor to poor reproductive health outcomes, the relationship between IPV and contraceptive use is unclear. Reproductive coercion (RC), a mechanism by which power is maintained over a partner through enforced reproductive behaviours, could be the missing link in understanding this relationship. However, there is limited understanding of RC and contraceptive use in sub-Saharan Africa.

Data Source and Methods: We used large-scale population based survey data from Ethiopia and examined the relationship between reproductive coercion and contraceptive use. In addtion, we also estimated the predictors of reproductive coercion. All models were estimated using multivariate logistic regression techniques.

Findings: Our findings suggest a strong negative association between RC and contraceptive use after adjusting for IPV and other factors, while emotional IPV was strongly predictive of RC.

Conclusion: Reproductive Coercion can be critical for understanding how controlling behaviours and violence manifest in the reproductive arena and impact family planning decision-making.
\end{abstract}

Keywords: Reproductive coercion, intimate partner violence, contraceptive use, Ethiopia

\section{Introduction}

Intimate partner violence (IPV) is a major contributor to poor reproductive health outcomes for women and girls worldwide. Women who experience IPV are more likely to experience unintended pregnancy (Miller et al 2010a; Fanslow et al 2008; Silverman et al 2007; Silverman et al 2010), repeated abortions, and miscarriages (Titilayo and Palamuleni 2015; Pallitto et al 2013; Silverman et al 2010; Alio, Nana, and Salihu 2009; Silverman et al 2007), increased risk of teenage pregnancy and sexually transmitted infections (Silverman et al 20II; Silverman, Mucci, and Hathaway 200I) as well as less likely to access health services (Udo, Doctor, Ahonsi 2018; Solanke, 2014). However, while the relationship between IPV and poor reproductive health outcomes is established, the pathways that explain this relationship are less well understood, especially with regard to how IPV influences contraceptive use behaviours. Reproductive coercion, or behaviour intended to maintain a male partner's power and control in a relationship by reducing female control over reproductive decisions by interfering with contraceptive use and decision-making around pregnancy through pregnancy coercion, contraceptive sabotage, and general lack of supportiveness in the reproductive arena, may be the missing link that could enhance our understanding of these connections (Silverman and Raj 2014; American College of Obstetricians and Gynecologists
2013; Moore, Frohwirth and Miller 2010). As such, reproductive coercion may be a crucial mechanism by which abuse in relationships influences use of contraception and ultimately leads to outcomes such as unintended pregnancy and repeated abortion.

While it is often assumed that IPV is likely to also influence contraceptive use, primarily because fear and/or threat of violence may diminish a woman's ability to refuse sex or negotiate family planning use, the relationship between IPV and contraceptive use appears to be more complex and context specific than this approach would suggest. At the theoretical level, the plausible pathways that connect IPV and contraceptive use also suggest mixed outcomes. For example, on the one hand, women may not want to risk contraceptive use because of fear of violence or they may use contraception to protect their future offspring (Hindin, Kishor, Ansara 2008). In addition, timing of violence vis-à-vis contraceptive use might also be important, something we do not currently understand well since much of the available evidence is based on cross-sectional data (Maxwell et al 20l5). The empirical evidence reflects this complexity; while some studies report reduced likelihood of contraceptive use among women who report IPV (Maxwell et al 2015; Stephenson, Jadhav and Hindin 2013; Hindin, Kishor and Ansara 2008), other studies have found similar or higher rates of contraceptive use among women reporting IPV as compared to 
those who do not report IPV (Stephenson, Jadhav and Hindin 2013; Alio et al 2009; Kishor and Johnson 2004). Yet other studies have found that although women subjected to abuse are more likely to have ever used contraception over their lifetime, they are more likely to use it inconsistently and less likely to use their preferred method (Kishor and Johnson 2004). Clearly, we have a long way to go in understanding the behavioural mechanisms that perpetuate lack of reproductive control among women and girls and provide a better understanding of the relationship between IPV and contraceptive use.

Evidence from the United States suggests that women and girls who report physical and sexual IPV are significantly more likely to experience reproductive coercion from male partners (American College of Obstetricians and Gynaecologists 2013; Miller et al 2010a; Moore, Frohwirth and Miller 2010). A rare study conducted exclusively among men reported that perpetrators of IPV were three times more likely to coerce a female partner to terminate or continue a pregnancy against her will (Silverman et al 2010). However, studies also suggest that reproductive coercion can also be present if other forms of violence are not present (Miller et al 2010a). Furthermore, studies have found that while reproductive coercion by itself is predictive of unintended pregnancy, it also interacts with other forms of IPV to elevate the risk of unintended pregnancy (Miller et al 20l0a). While most of the current literature on reproductive coercion is focused on the United States, new evidence from developing countries such as Côte d'Ivoire, Jordan and Pakistan is shedding new light on the phenomenon (Falb et al 20I4; McCleary-Sills 20I3; Zakar et al 2012). These studies indicate that in addition to their male partners, women might also be subjected to reproductive coercion from family members of their male partner, such as their motherin-law, in some contexts (Clark et al 2010; Gupta et al 20 I2; Raj et al 2006).

Despite sub-Saharan Africa reporting some of the highest levels of IPV (McCloskey et al 2016) and lowest levels of contraceptive use (Gribble and Haffey 2008), there is very limited evidence exploring the relationship between IPV, reproductive coercion, and contraceptive use, and other reproductive health outcomes in this context. A study based on analysis of Demographic and Health Surveys (DHS) conducted between 2003-2006 in 6 sub-Saharan African countries - Cameroon, Kenya, Malawi, Rwanda, Uganda, and Zimbabwe - found higher contraceptive use among women who reported sexual and physical IPV (Alio et al 2009). However, no study to our knowledge has conducted an in-depth analysis of the influence of different forms of IPV, including reproductive coercion, on contraceptive use in this context. To fill this gap, we used data from a largescale population based study in Ethiopia and examined the linkages between reproductive coercion, IPV, and contraceptive use. In addition, we also accounted for other relationship factors, such as age difference between spouses and polygamous marriages, that are known to affect male power within marital relationships in the analysis. Furthermore, to improve robustness of our results, we also adjust for the influence of community norms around reproductive coercion and contraceptive use. Finally, given the limited understanding of reproductive coercion in this context, we also explore the predictors of reproductive coercion.

\section{Data source and methods \\ Data}

Data for this study was derived from a multi-country study aimed at estimating the economic costs of child marriage. Ethical approval was obtained both at the global and local level. At the global level, review was provided by the Institutional Review Board from the International Center for Research on Women, while in Ethiopia the review was provided by the National Research Ethics Review Committee. The study surveyed ever-married women in the age range of 18-45 years. In Ethiopia, a total of 4,149 women were interviewed in nine regions and one town administration of the country. A three-stage stratified sampling approach was used to develop the sample. First, within each region, districts were selected by probability proportional to size, followed by random selection of counties and villages. A household census was then conducted within each village, followed by random selection of 25 households. If a woman did not consent to participate, the field team selected another eligible woman from the same household or an adjacent household. In cases where a family head had multiple wives, only one randomly selected wife was interviewed. The women's questionnaire collected detailed information on the woman's demographic background, health history (including mental health), and experience with intimate partner violence, including reproductive coercion. In addition, the household questionnaire collected information on household wealth, demographic and health information of household members.

\section{Key measures}

Current contraceptive use was measured as a dummy variable based on women's report of using a modern method of contraception at the time of the interview. The methods included to construct this 
variable were injectables, implants, intra-uterine device (IUD), pill, condoms and female sterilization.

Reproductive coercion was measured with a series of statements: (I) In the past 12 months, has your current husband/partner told you not to use any birth control? (2) In the past 12 months, has your current husband/partner hurt you physically because you did not agree to get pregnant? (3) In the past 12 months, has your current husband/partner taken your birth control (such as pills) away from you or kept you from going to the clinic to get birth control so that you would get pregnant?" (4) Does your partner support your decision about when or if you want to become pregnant? Very few women reported being physically hurt by their partner for refusing to become pregnant $(2.25 \%)$, while almost $10 \%$ of the women reported being told by their partner to not use any birth control, about 5\% reported their partners kept them from going to the clinic to get birth control or took their birth control away from them, and half the women reported not feeling supported on if and when to become pregnant. The items had high internal consistency (Cronbach's Alpha $=0.95$ ). Women were coded as having experience reproductive coercion if they answered 'yes' to any of the first three statements or 'no' to the final statement.

Intimate partner violence (IPV) measures were adapted from the World Health Organization's MultiCountry Study on Women's Health and Domestic Violence Against Women (Garcia-Moreno et al 2005). The survey categorized violence into physical, sexual and emotional domains, with respondents asked to report IPV experienced in the 12 months prior to the survey as well as IPV experienced before that point, providing measures of both recent and lifetime experience. To measure physical IPV, the women were asked if their husband or partner does/did the following: slapped or had something thrown at them that could hurt them, pushed or shoved them, hit them with their fist or something else that could hurt, kicked, dragged or beaten or choked or burnt them on purpose, threatened to use or used a gun, knife or other weapon against her. Similarly, for sexual IPV, they were asked if their husband/partner does/did the following: physically forced them to have sexual intercourse when she did not want to, had sexual intercourse when they did not want to because they were afraid of what their partner might do if they refused, or were forced to do anything sexual that they did not want to do. Finally, for emotional IPV, the women were asked if their husband or partner had: insulted or made them feel bad about themselves, belittled or humiliated them in front of other people, scared or intimidated them on purpose, e.g. by the way male partners looked at them, by yelling or smashing things, or threatened to hurt someone they cared about. For every indicator of physical, sexual and emotional IPV, the woman was asked if she had experienced the event once, a few or many times in the 12 months preceding the survey or prior to the preceding 12 month period. A positive answer to any of the indicators was coded as evidence of physical IPV, sexual IPV or emotional IPV. Separate and combined indicators of physical, sexual and emotional violence were created to indicate life-time experiences of IPV (combined last 12 months and before the last 12 months).

Furthermore, in addition to IPV and reproductive coercion, two additional variables - spousal age difference and Polygamy - were included in the analysis that are known to capture power differentials between spouses. To generate spousal age difference, the wife's age was first subtracted from her partner's age. Polygamy was measured as a dummy variable that differentiated between polygamous and monogamous marriages. Finally, community level measures for reproductive coercion and contraceptive use were generated to capture some of the normative influences on these behaviours. To construct, measures of contraceptive use and reproductive coercion were aggregated from individual responses within each primary sampling unit, excluding the index respondent's response.

\section{Other background variables}

A range of standard socio-demographic, household and couple-level variables known to influence reproductive coercion and contraceptive use were included in the analysis, such as the respondents' age, education, number of children, household wealth and religion. Age was measured as a continuous variable denoting age in years. Dummy variables were created to measure school attendance and employment status. Number of children was calculated using the respondents' answers to a series of questions on her childbirth history. Household wealth was constructed through a principal components analysis of household assets, and a battery of questions on housing characteristics such as ownership of consumer items and type of dwelling. The index score was then used to divide the households into the quintiles of poorest, poor, middle, richer and richest households. Finally, place of residence indicating urban versus rural residence and regional dummies representing the different regions of Ethiopia were also included in the models.

\section{Statistical analysis}

We conducted multivariate logistic regressions with contraceptive use as the dependent variable, and 
reproductive coercion and IPV as the key independent variables. In addition, we included critical variables such as spousal age difference and monogamous versus polygamous marriage in the model, thereby allowing for a direct estimation of the effect of experience of reproductive coercion on current contraceptive use. Finally, we adjusted for standard background variables, including community norms around contraceptive use and reproductive coercion. In addition, we conducted a multivariate logistic regression to estimate the predictors of reproductive coercion. All analyses were performed in Stata 14. Data were adjusted to account for the complex survey design, including clustering of data.

\section{Results}

Since the focus of our analysis was reproductive coercion, which was measured for the last 12 months, we restricted the sample to currently married/partnered women $(n=3870)$. In addition, given the focus on contraceptive use, we restricted the sample to currently non-pregnant women who did not report an intention to become pregnant in the near future, which resulted in an analytical sample of women.

\section{Table 1A}

Descriptive Statistics of Key Variables by Study Population for the Determinants of Reproductive Coercion Analysis $(\mathbf{n}=\mathbf{3 8 7 0})^{1}$

\begin{tabular}{lc}
\hline VARIABLES & \% or mean (SD) 1 \\
\hline Age in Years & $29.39(7.35)$ \\
Ever attended School & 35.88 \\
Currently Working & 13.28 \\
No of Children & $3.7(2.37)$ \\
Polygamous Marriage & 8.64 \\
Spousal Age Difference & $7.12(5.56)$ \\
Physical and Sexual Violence & 29.97 \\
Emotional Violence & 25.10 \\
Reproductive Coercion & 57.61 \\
\hline
\end{tabular}

Sample excludes women currently not in partnership- delete as already described under methods

\section{Table 1B}

Descriptive Statistics of Key Variables by Study Population for the Contraceptive Use Analysis $(n=2380)^{1}$

\begin{tabular}{lc}
\hline VARIABLES & \% or mean (SD) 1 \\
\hline Age in Years & $38.69(7.37)$ \\
Ever attended School & 13.21 \\
Currently Working & 12.97 \\
No of Children & $3.7(2.37)$ \\
Polygamous Marriage & 8.01 \\
Spousal Age Difference & $7.02(5.56)$ \\
Intimate Partner Violence & 31.31 \\
Reproductive Coercion & 58.11 \\
Contraceptive Use & 38.69 \\
\hline
\end{tabular}

${ }^{1}$ Sample excludes currently pregnant women and those who are trying to become pregnant

as well as, women currently not in partnership- see above

Table IA provides descriptive statistics of key study variables for the analysis focused on the determinants of reproductive coercion, while Table IB contains descriptive statistics for key study variables for the sample eligible for the contraceptive use analysis. As shown in Table IA, the women on average were 29.39 (SD: 7.35 ) years old and $35.88 \%$ had attended school. About $30.0 \%$ of the women had experienced physical and sexual IPV, while $25.10 \%$ had experienced emotional IPV over their lifetime, and $57.61 \%$ had faced reproductive coercion. On the other hand, on average, respondents who had ever used contraceptives were older, averaging 38.69 (SD: 7.37) years (Table IB). Levels of schooling and work participation were low, $13.21 \%$ of the women had attended school and $12.97 \%$ were currently working. A little over a third of the women were using contraceptives (38.69\%), while $31.31 \%$ had experienced physical and sexual IPV over their lifetime and $58.11 \%$ had faced reproductive coercion in the last 12 months preceding the survey. 


\section{Table 2}

Distribution of Contraceptive Use by Key Study

Variables (n=2380) *

\begin{tabular}{lcc}
\hline VARIABLES & \multicolumn{2}{c}{ \% or Mean (SD) } \\
\hline Age in Years & Users & Non-users \\
Schooling & $28.71(0.19)$ & $31.18(0.17)$ \\
Currently Working & 53.65 & 46.35 \\
No of Children & 44.52 & 55.48 \\
Polygamous Marriage & $3.3(0.05)$ & $3.8(0.05)$ \\
Spousal Age Difference & 5.27 & 11.81 \\
Intimate Partner Violence & $6.76(4.86)$ & $7.65(6.22)$ \\
Reproductive Coercion & 27.68 & 33.06 \\
\hline
\end{tabular}

* Sample excludes currently pregnant women and those who are trying to become pregnant as well as, women currently not in partnership

Table 2 provides the bi-variate distribution of contraceptive use by key study variables. When comparing contraceptive users to non-users, they were on average younger than non-users with smaller age gaps with their partners, more likely to have attended school, less likely to be working, be in a polygamous marriage, or experience reproductive coercion or IPV.

\section{Table 3}

Multivariate Logistic Regression Analysis Examining

the Association of Reproductive Coercion with Contraceptive Use $(\mathbf{n}=\mathbf{2 3 8 0})^{2}$

VARIABLES

Reproductive Coercion

Age in Years

Age Squared

Schooling

Currently Working

No of Children No children (ref)

\section{1-2 Children}

\section{3-5 Children}

More than 5 Children

Polygamous Marriage

Spousal Age Difference in Years

Intimate Partner Violence

Household Wealth (Ref Group: Poorest)

Poorer

Middle

Richer
$(1.01-1.96) * *$

1.42

0.77

(0.61 - 0.97) **

1.26

$(1.11-1.43) * * *$

0.99

$(0.99-1.00) * * *$

1.49

$(1.23-1.82) * * *$

1.14

(0.81 - 1.61)

2.81

$(1.90-4.16) * * *$

3.48

(2.27 - 5.35) ***

2.71

$(1.66-4.43) * * *$

0.67

$(0.43-1.03) *$

0.98

$(0.96-1.00) * *$

0.78

$(0.63-0.97) * *$

$(1.00-2.03) *$

1.38

$(0.99-1.91) *$ 
Richest

Urban (ref=rural)

Reproductive Coercion Cluster Level

Contraceptive Use Cluster Level
1.40

$(1.04-1.89) * *$

1.07

$(0.87-1.30)$

0.86

$(0.45-1.64)$

16.28

$(8.40-31.56) * * *$

$* * * \mathrm{p}<0.001, * * \mathrm{p}<0.05, * \mathrm{p}<0.1$

${ }^{1}$ Sample also adjusted for religion, region and sampling weights

${ }^{2}$ Sample excludes currently pregnant women and those who are trying to become pregnant as well as, women currently not in partnership

Table 3 presents results from the multivariate regression analysis estimating the association between reproductive coercion, IPV, and contraceptive use, while Table 4 provides results from the analysis examining the predictors of reproductive coercion. In the multivariate analysis, reproductive coercion significantly reduced the odds of contraceptive use (OR: $0.77, \mathrm{Cl}: 0.6 \mathrm{I}-0.97$ ) even after adjusting for IPV and other key factors (Table 3). Lifetime experience of IPV also significantly reduced the odds of contraceptive use (OR: $0.78, \mathrm{Cl}$ : 0.63-0.97). Among other relationship factors, while a larger spousal age difference reduced the odds of contraceptive use, polygamous marriage increased the odds of use (Table 3). In addition, community norms around contraceptive use were highly predictive of contraceptive use (Table 3), with those women living in communities with higher use much more likely to be using themselves.

In terms of predictors of reproductive coercion, while having a larger number of children was protective, possibly reflecting the greater pressure on nulliparous women to bear children, being Muslim and older increased the risk of reproductive coercion (Table 4). Also, interestingly, while experience of physical or sexual IPV did not predict reproductive coercion, emotional IPV was predictive of reproductive coercion. Presence of emotional IPV increased the odds of reproductive coercion by $4 \mathrm{I}$ $\%$. However, factors such as woman's education, work status and household wealth were not predictive of reproductive coercion.

\section{Table 4}

Multivariate Logistic Regression Analysis Examining the Predictors of Reproductive Coercion $(n=3870)^{2}$

\begin{tabular}{|c|c|}
\hline VARIABLES & $\mathrm{OR}(\mathrm{CI})$ \\
\hline \multicolumn{2}{|c|}{ Woman's Age (Ref Group: $15-20$ years) } \\
\hline $20-24$ years & $\begin{array}{c}0.96 \\
(0.72-1.29)\end{array}$ \\
\hline 25-29 years & $\begin{array}{c}1.33 \\
(0.95-1.85) *\end{array}$ \\
\hline 30-34 years & $\begin{array}{c}1.42 \\
(0.96-2.08) *\end{array}$ \\
\hline 35-39 years & $\begin{array}{c}1.74 \\
(1.19-2.56) * *\end{array}$ \\
\hline 40-45 years & $\begin{array}{c}1.94 \\
(1.27-2.97) * *\end{array}$ \\
\hline Schooling & $\begin{array}{c}1.04 \\
(0.88-1.22)\end{array}$ \\
\hline Currently Working & $\begin{array}{c}0.89 \\
(0.68-1.18)\end{array}$ \\
\hline \multicolumn{2}{|l|}{ No of Children } \\
\hline 1-2 Children & $\begin{array}{c}0.88 \\
(0.68-1.13)\end{array}$ \\
\hline 3-5 Children & $\begin{array}{c}0.77 \\
(0.60-1.00) *\end{array}$ \\
\hline More than 5 Children & $\begin{array}{c}0.67 \\
(0.46-0.95) * *\end{array}$ \\
\hline
\end{tabular}

Religion (Ref Group: Muslim) 
Household Wealth (Ref Group: Poorest)

Poor

Middle

1.34

$(1.04-1.72) * *$

1.11

$(0.85-1.44)$

0.98

$(0.76-1.26)$

0.96

Richest

$(0.72-1.28)$

\footnotetext{
$* * * \mathrm{p}<0.001, * * \mathrm{p}<0.05, * \mathrm{p}<0.1$

${ }^{1}$ Also adjusted for household wealth, urban/rural residence, region \& sampling weights

${ }^{2}$ Sample excludes women currently not in partnership
}

\section{Discussion}

This study found a negative statistically significant relationship between reproductive coercion and contraceptive use. These results remained robust even after adjusting for IPV as well as other relationship factors that determine power differentials between partners such as spousal age difference and polygamy. The models were also adjusted for community norms around contraceptive use as well as reproductive coercion. The findings suggest a direct relationship between reproductive coercion and contraceptive use, broadening understanding of the mechanisms that link male partner's reproductive control over the couple's contraceptive use and fertility decisions in the Ethiopian context. These connections highlight the opportunities family planning programs and policies can utilize to reduce the burden of IPV. Miller et al $(2010 b)$ suggest that reproductive health professionals are in a critical position to reach women who are in abusive relationships and recommend that they should be trained and provided with the tools to screen and help women facing reproductive coercion, so that they are able to achieve their reproductive goals (Miller et al 20I0b). While we are at the initial stages of understanding reproductive coercion in Ethiopia, our results provide preliminary evidence of the mechanisms that connect IPV with family planning use in this context and indicate the potential role health systems could play in reducing the risk of IPV in this context.

Among the predictors of reproductive coercion, although the association of age and parity with reproductive coercion was in the expected direction, physical and sexual IPV were not predictive of reproductive coercion, while emotional IPV was highly predictive of reproductive coercion. This perhaps suggests that reproductive coercion might be present even in situations where more extreme forms of IPV are not present, or may be codetermined by features of spousal relations that also determine emotional abuse. This finding highlights the need to educate women on male partner's controlling behaviours, since some women, especially younger women, may not even recognize these behaviours as being coercive and may be more susceptible to accept their male partner's wishes because of pressure from the extended family or their own ambivalence regarding their pregnancy. Introduction of comprehensive sexuality education curricula that integrates discussion on partner violence and reproductive coercion, helps women recognize linkages between unhealthy relationships and constrained reproductive autonomy and provides them with the tools and information needed to strategize and protect their sexual and reproductive health can go a long way in offsetting some of these negative outcomes (Miller et al 20l Ob).

Our study has several limitations that need to be considered while interpreting the results. A major limitation is the cross-sectional design, which makes it hard to ascertain the temporal order of events. Moreover, while our reproductive coercion measures have been validated in other studies and allow for cross-cultural comparison, these studies were primarily conducted in the United States and so might be missing context-specific information. There is need for formative research in Ethiopia to really 
understand how reproductive coercion manifests, so that more context-specific measures can be developed. Despite these limitations, our study has several strengths. This is one of the earliest studies identifying the presence of reproductive coercion in the Ethiopian context and its relationship with contraceptive use, providing a new lens towards understanding contraceptive decision-making. We also use a large-scale population-based survey to explore these connections which is representative of the Ethiopian population.

\section{Conclusion}

Reproductive coercion can be critical for understanding how controlling behaviours and violence operate in the reproductive arena and impact family planning decision-making. However, more research is needed to understand the nuances of how reproductive coercion manifests in different settings to develop context specific measures. Reproductive health programs and policies could utilize this knowledge to reduce violence and improve family planning use. Reproductive health care providers can play a critical role in the prevention and treatment of reproductive coercion by screening women and connecting severe cases to necessary services as well as empowering women to identify and strategize around reproductive coercion so as to retain their reproductive autonomy and accomplish their reproductive goals.

\section{Acknowledgement:}

Authors are grateful for support from the Bill \& Melinda Gates Foundation for funding the research study from which data was obtained for the analysis presented in this paper and William and Flora Hewlett Foundation for supporting the writing of this manuscript.

\section{References}

Alio, A., Daley, E., Nana, P., Duan, J. and Salihu, H. (2009). Intimate partner violence and contraception use among women in Sub-Saharan Africa. International Journal of Gynecology \& Obstetrics, I07(I), pp.35-38.

Alio, A., Nana, P. and Salihu, H. (2009). Spousal violence and potentially preventable single and recurrent spontaneous fetal loss in an African setting: cross-sectional study. The Lancet, 373(9660), pp.318-324.

Solanke, B.L. (20|4). Association between intimate partner violence and utilization of maternal health services in Nigeria. African Population Studies, 28 (2), 933.

'Clark, C., Silverman, J., Shahrouri, M., EversonRose, S. and Groce, N. (20I0). The role of the extended family in women's risk of intimate partner violence in Jordan. Social Science \& Medicine, 70(I), pp.I44-I5I.

Falb, K., Annan, J., Kpebo, D., \& Gupta, J. (20|4). Reproductive Coercion and Intimate Partner Violence among Rural Women in Côte d'lvoire: A Cross-sectional Study. African Journal of Reproductive Health / La Revue Africaine De La Santé Reproductive, 18(4), 6I-69.

Fanslow J, Whitehead A, Silva M, Robinson E (2008). Contraceptive use and associations with intimate partner violence among a population-based sample of New Zealand women. The Australian and New Zealand Journal of Obstetrics and Gynaecology, 48(I), pp.83-89.

García-Moreno C, Jansen HA, Ellsberg M, Heise L, Watts C. (2005). World Health Organization multi-country study on Women's health and domestic violence against women: initial results on prevalence, health outcomes and women's responses. Geneva, Switzerland: WHO.

Gribble, J. and Haffey, J. (2008). Reproductive Health in Sub-Saharan Africa. [online] Washington DC: Population Reference Bureau. Available at: http://www.prb.org/pdf08/reproductivehealth_su bsaharanafrica.pdf. Accessed on II/05/2017.

Gupta, J., Falb, K., Kpebo, D. and Annan, J. (20/2). Abuse from in-laws and associations with attempts to control reproductive decisions among rural women in Côte d'lvoire: a cross-sectional study. BJOG: An International Journal of Obstetrics \& Gynaecology, I I9(9), pp. I058-1066.

Hindin, Michelle J., Sunita Kishor, and Donna L. Ansara. (2008). Intimate Partner Violence among Couples in 10 DHS Countries: Predictors and Health Outcomes. DHS Analytical Studies No. 18. Calverton, Maryland, USA: Macro International Inc.

Kishor, Sunita and Kiersten Johnson. (2004). Profiling Domestic Violence - A Multi-Country Study. Calverton, Maryland: ORC Macro.

Maxwell, L., Devries, K., Zionts, D., Alhusen, J. and Campbell, J. (2015). Estimating the Effect of Intimate Partner Violence on Women's Use of Contraception: A Systematic Review and MetaAnalysis. PLOS ONE, I0(2), p.e0I I 8234.

McCleary-Sills J. (20/3). Jordanian social norms and the risk of intimate partner violence and limited reproductive agency. J Int Womens Stud, I 4(2): I 2-29.

McCloskey, L., Boonzaier, F., Steinbrenner, S. and Hunter, T. (2016). Determinants of Intimate Partner Violence in Sub-Saharan Africa: A Review of Prevention and Intervention Programs. Partner Abuse, 7(3), pp.277-3I5. 
Miller, E., Decker, M., McCauley, H., Tancredi, D., Levenson, R., Waldman, J., Schoenwald, P. and Silverman, J. (20I0a). Pregnancy coercion, intimate partner violence and unintended pregnancy. Contraception, 8I (4), pp.3।6-322.

Miller, E., Jordan, B., Levenson, R. and Silverman, J. (20IOb). Reproductive coercion: connecting the dots between partner violence and unintended pregnancy. Contraception, 8I (6), pp.457-459.

Moore, A., Frohwirth, L. and Miller, E. (20I0). Male reproductive control of women who have experienced intimate partner violence in the United States. Social Science \& Medicine, 70(I I), pp. I737-I 744.

Pallitto CC, Garci'a-Moreno C, Jansen H, Heise L, Ellsberg M, et al. (20/3) Intimate partner

violence, abortion, and unintended pregnancy: Results from the WHO Multi-Country Study on

Women's Health and Domestic Violence. Int J Gynaecol Obstet 120: 3

Raj, A., Livramento, K., Santana, M., Gupta, J. and Silverman, J. (2006). Victims of Intimate Partner

Violence More Likely to Report Abuse From In-Laws. Violence Against Women, I2(10), pp.936-949.

Reproductive and sexual coercion. (20/3). Committee Opinion No. 554. American College of Obstetricians and Gynecologists. Obstet Gynecol; |2|:4| I-5.

Silverman, J., Raj, A., Mucci, L. and Hathaway, J. (200I). Dating Violence Against Adolescent Girls and Associated Substance Use, Unhealthy Weight Control, Sexual Risk Behavior, Pregnancy, and Suicidality. JAMA, 286(5), p.572.

Silverman, J., Gupta, J., Decker, M., Kapur, N. and Raj, A. (2007). Intimate partner violence and unwanted pregnancy, miscarriage, induced abortion, and stillbirth among a national sample of Bangladeshi women. BJOG: An International
Journal of Obstetrics \& Gynaecology, II4(10), pp. I 246-I 252.

Silverman JG, Decker MR, McCauley HL, Gupta J, Miller E, et al. (20I0) Male perpetration of intimate partner violence and involvement in abortions and abortion-related conflict. Am J Public Health, 100: |4I5-14I7.

Silverman, J. and Raj, A. (20|4). Intimate Partner Violence and Reproductive Coercion: Global

Barriers to Women's Reproductive Control. PLoS Medicine, II(9), p.el00I 723.

Solanke, B.L., 20I4. Association between intimate partner violence and utilisation of maternal health services in Nigeria. Etude de la Population Africaine, 28(2), p.933.

Silverman, J., McCauley, H., Decker, M., Miller, E., Reed, E. and Raj, A. (20II). Coercive Forms of Sexual Risk and Associated Violence Perpetrated by Male Partners of Female Adolescents. Perspectives on Sexual and Reproductive Health, 43(I), pp.60-65.

Stephenson, R., Jadhav, A. and Hindin, M. (20/3). Physical Domestic Violence and Subsequent Contraceptive Adoption Among Women in Rural India. Journal of Interpersonal Violence, 28(5), pp. 1020-1039.

Titilayo, A. and Palamuleni, M.E., 2015. Spousal violence and unwanted fertility in Malawi. Etude de la Population Africaine, 29(2).

Udo, I.E., Doctor, H.V., \& Ahonsi, B.A. (20/8). Intimate partner violence and uptake of HIV testing and STI treatment among married women in Nigeria. African Population Studies, 32 (I).

Zakar, R., Zakar, M., Mikolajczyk, R. and Krämer, A. (20/2). Intimate partner violence and its association with women's reproductive health in Pakistan. International Journal of Gynecology \& Obstetrics, $\quad 117(1)$, pp. I0-I4. 